\title{
Depletion forces due to image charges near dielectric discontinuities
}

\author{
Robin A. Curtis ${ }^{\mathrm{a}}$, Leo Lue $\mathrm{L}^{\mathrm{b}, *}$ \\ ${ }^{a}$ School of Chemical Engineering and Analytical Science, The University of Manchester \\ Oxford Road, Manchester M13 9PL, United Kingdom \\ ${ }^{b}$ Department of Chemical and Process Engineering, University of Strathclyde \\ James Weir Building, 75 Montrose Street, Glasgow G1 1XJ, United Kingdom
}

\begin{abstract}
The depletion force is an effective inter-particle attractive interaction that is entropically driven by the exclusion of co-solvent molecules. For large co-solvents, such as polymers, the exclusion is primarily driven by excluded volume interactions. However, the exclusion of co-solvents, such as electrolytes, can be caused by other mechanisms. In this review, we summarize the literature on interparticle depletion forces that arise from repulsive image-charge forces between low-dielectric particles and electrolytes. In particular, we emphasize the results from a variational perturbation theory for describing the salting-out behavior observed in moderately concentrated salt solutions. The theory predicts an unscreened force with a range given by the Bjerrum length and a magnitude proportional to the osmotic pressure of the salt solution. The force becomes significant at the same salt concentration where salting-out behavior is typically observed.
\end{abstract}

Keywords: depletion force, dielectric, electrolyte, colloids

\section{Introduction}

The depletion force was originally used to describe the attraction between colloidal particles induced by the addition of small polymer molecules [1. In

\footnotetext{
${ }^{*}$ Corresponding author

Email address: leo.lue@strath.ac.uk ( Leo Lue )
} 
this case, the force is driven by polymer exclusion from the colloidal particles.

${ }_{5}$ The excluded volume of the colloids restricts the space available to the polymer molecules. The net exclusion volume decreases as colloids approach each other, giving rise to a net inter-colloid attractive force, with a range and magnitude determined by the polymer size and the polymer osmotic pressure, respectively.

Excluded volume interactions are one mechanism that gives rise to an effective inter-colloid depletion force. However, any situation where co-solvents (e.g., polymers) are driven from the surface of a colloidal particle can lead to an entropically driven attraction between the colloids. In this work, we review and discuss the depletion of ions from a dielectric interface due to image-charge interactions. This depletion of ions leads to an effective attraction between larger

15 colloidal particles. We discuss the similarities and differences between depletion forces driven by image-charge forces and by excluded volume interactions.

\section{Image-charge interactions}

\subsection{Planar interfaces}

Consider a planar interface located at $z=0$ which separates two mediums with dielectric constants equal to $\varepsilon^{\prime}$ and $\varepsilon$ as shown schematically in Fig. 1. The medium to the left corresponds to a particle, which is inaccessible to electrolytes. The right side is referred to as the solvent and contains electrolytes (co-solvent). A point charge with magnitude $q$ located a distance $d$ from the interface will induce a surface charge due to the difference in polarizabilities between the two media. For an isolated planar interface, the potential of the induced surface charge is identical with that of a point charge of magnitude $q^{\prime}=\Delta q$, where $\Delta=\left(\varepsilon-\varepsilon^{\prime}\right) /\left(\varepsilon+\varepsilon^{\prime}\right)$, located at $z=-d$, in the absence of the dielectric discontinuity [2]. The interaction energy $u(\mathbf{r})$ of the ion with the induced surface charge is

$$
\beta u(\mathbf{r})=\frac{\Delta l_{B}}{2 z},
$$

where $\beta=\left(k_{B} T\right)^{-1}, k_{B}$ is the Boltzmann constant, $T$ is the absolute temperature of the system, and $l_{B}=\beta q^{2} / \varepsilon$ is the Bjerrum length. If $q$ is the elementary 
charge, then $l_{B} \approx 7 \AA$ for water at $25^{\circ} \mathrm{C}$.

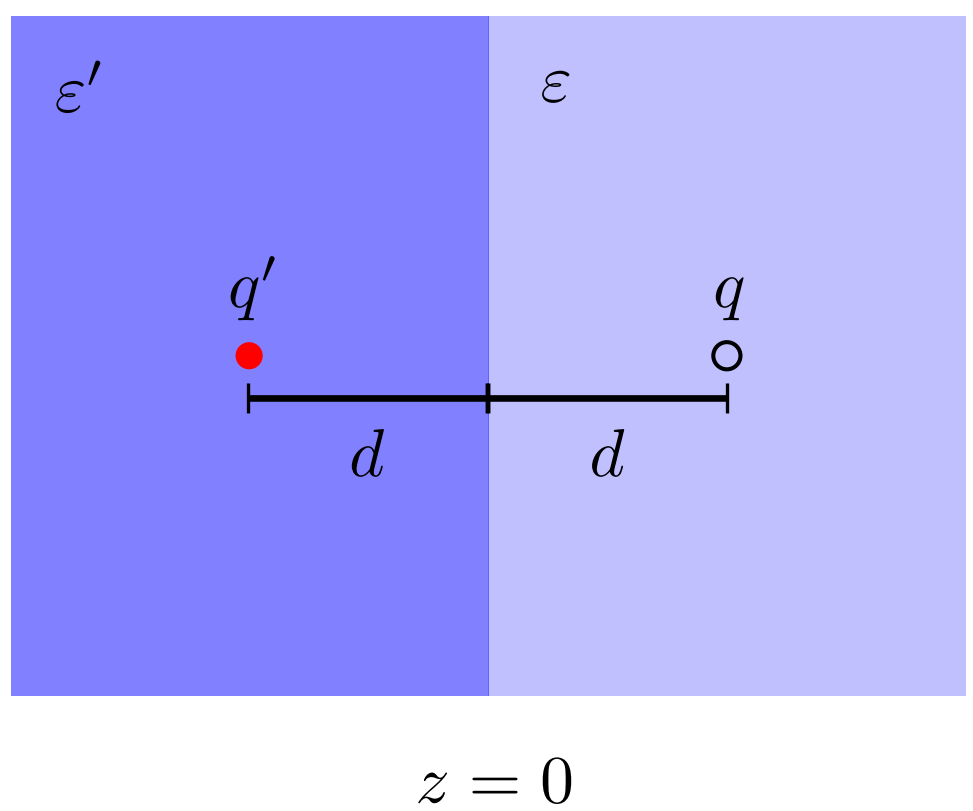

Figure 1: Schematic diagram of a point charge near a planar dielectric interface.

There is an effective ion-interface attraction when the dielectric constant of the particle is higher than that of the solvent (i.e. $\varepsilon^{\prime}>\varepsilon$ ). Conversely, if the dielectric constant of the particle is lower (i.e. $\varepsilon^{\prime}<\varepsilon$ ), then the ion is repelled 25 from the interface. The strength of the interaction varies as the square of the ion valency but is independent of the sign of the ion charge. The image force causes a layer of ion depletion about the interface, defined by a depletion length of roughly $l=\Delta l_{B} / 2$, the distance at which the image-charge interaction is of order $k_{B} T$.

\subsection{Spherical geometries}

The strength of the image-charge interaction depends on the geometry of the dielectric interface. As an illustrative example, consider a single sphere with a dielectric constant $\varepsilon^{\prime}$ embedded in a solvent of dielectric constant $\varepsilon$. The presence of charge induces a surface charge on the dielectric interface. For a 
point charge located a distance $r$ from the center of the dielectric sphere, the potential generated by the induced surface charge can be represented by the sum of a point charge of magnitude $q_{K}=\Delta q(R / r)$ located at a distance $r_{K}=R^{2} / r$ from the center of the sphere and a line charge stretching from the center of the sphere to $r_{K}$ with a linear charge density [3, 4, 5, 6]

$$
\lambda_{K}(x)=-\Delta q \zeta\left(\frac{r_{K}}{x}\right)^{1-\zeta}
$$

where $x$ is the distance from the center of the sphere, $\eta=\varepsilon^{\prime} / \varepsilon$, and $\zeta=(1+\eta)^{-1}$.

In the limit that $\varepsilon^{\prime} \gg \varepsilon$ or $\varepsilon^{\prime} \ll \varepsilon$, the expressions for the Green's function and ion self energy can be written in closed form [3, 4, 6. The self energy in this case is exactly given by

$$
\beta u(\mathbf{r})=\frac{l_{B}}{R}\left[\frac{R^{2}}{r^{2}-R^{2}}+\ln \left(1-R^{2} / r^{2}\right)\right] .
$$

The image-charge interaction approaches that of a plane when the distance from the point charge to the surface is much less than the sphere radius. In general, the image-charge interaction for the dielectric sphere is weaker than that for the plane.

When a point charge is inside a sphere of dielectric constant $\varepsilon$ that is surrounded by a medium of dielectric constant $\varepsilon^{\prime}$, the image charge can again be represented as a point charge and a line charge. The point charge has magnitude $q_{K}=\Delta q R / r$ and is located at a distance $r_{K}=R^{2} / r$ from the center of the sphere. The line charge stretches from the point $r_{K}$ outside the sphere to infinity and has a linear charge density given by Eq. (2).

\subsection{Other geometries}

For other more complex geometries, the potential generated by the induced charge cannot be easily represented in terms of simple image charge distributions. The key quantity in these electrostatic problems is the Green's function $G\left(\mathbf{r}, \mathbf{r}^{\prime}\right)$ of the Poisson equation [2]

$$
-\frac{1}{4 \pi} \nabla \cdot\left[\varepsilon(\mathbf{r}) \nabla G\left(\mathbf{r}, \mathbf{r}^{\prime}\right)\right]=\delta^{d}\left(\mathbf{r}-\mathbf{r}^{\prime}\right),
$$


where $\delta^{d}\left(\mathbf{r}-\mathbf{r}^{\prime}\right)$ is the $d$-dimensional Dirac delta function.

The Green's function gives the electric potential of a point charge under a given set of boundary conditions. The potential generated by a general charge distribution $Q(\mathbf{r})$ is given by

$$
\phi(\mathbf{r})=\int d \mathbf{r}^{\prime} G\left(\mathbf{r}, \mathbf{r}^{\prime}\right) Q(\mathbf{r}) .
$$

The Green's function can be represented as

$$
G\left(\mathbf{r}, \mathbf{r}^{\prime}\right)=G_{\text {free }}\left(\mathbf{r}, \mathbf{r}^{\prime}\right)+\delta G\left(\mathbf{r}, \mathbf{r}^{\prime}\right)
$$

where $G_{\text {free }}\left(\mathbf{r}, \mathbf{r}^{\prime}\right)=\left(\varepsilon\left|\mathbf{r}-\mathbf{r}^{\prime}\right|\right)^{-1}$ is the Green's function in the absence of any

45 dielectric bodies, and $\delta G\left(\mathbf{r}, \mathbf{r}^{\prime}\right)$ represents the influence of any dielectric bodies in the system.

Although analytical expressions do exist for general geometries, most require intensive computation, and, consequently, have limited value to direct simulations of systems containing electrolytes. The key challenge in the simulation and theoretical analysis of problems near dielectric interfaces is the development of new analytical expressions or algorithms for rapidly calculating the Green's function. Recently, several computational algorithms have been developed that efficiently evaluate the electrostatic interaction for arbitrary distributions of the dielectric constant. [7, 8, 9, 10, 11]. For collections of spheres, a fairly efficient quadrature method to integrate the image-charge interactions has been proposed [12, 13. In order to account for the dispersion interaction, however, further work is required to numerically evaluate the determinant of the Green's function.

\section{Electrolytes near dielectric bodies}

\subsection{Ion depletion}

For a symmetric electrolyte solution near a neutral dielectric surface, the average cation charge density will be identical to the average anion charge density. As a consequence, there will be no charge separation, and the system will 
be everywhere neutral on average. Within mean field theories, the ion density profiles are considered only in an average manner, and, consequently, there will be no repulsion of ions from a low-dielectric interface. Thus, one has to go beyond mean field theories, such as the Poisson-Boltzmann theory, and allow for fluctuations in ion densities to account for ion depletion.

In early theoretical approaches [14, 15], the image-charge interaction was included explicitly by using an additional one-body interaction. More recent theories account for correlations between ions, as well as between ions and the dielectric interface, using various methods, such as the self-consistent BornGreen-Yvon equation [16, 17, 18, the hypernetted-chain approximation [19, 20], loop expansions [21, 22, 23], variational perturbation theory [24, 25, strong cou75 pling expansions [26], among many others. These correlations lead to deviations from the mean ion profiles.

Applying these approaches to describe the behavior of small colloids such as proteins or spherical micelles requires determining the effect of curvature on the interfacial behavior. This has been taken into account using a variational perturbation theory. Figure 2 shows the ion density profile, as calculated using variational perturbation theory [24, around a sphere of radius $R$ and dielectric constant $\varepsilon^{\prime}$ immersed in a symmetric electrolyte (the co-solvent) dissolved in a solvent with dielectric constant $\varepsilon$, where $\varepsilon^{\prime} \ll \varepsilon$. The electrolyte concentration is such that the bulk screening length is $\kappa^{-1}=10 l_{B}$. The effect of curvature ${ }_{85}$ on the density profiles of a symmetric electrolyte about a spherical interface are shown in Fig. 2, For this calculation, the ratio of dielectric constants for the spherical particle over the solvent is taken as $\varepsilon^{\prime} / \varepsilon \ll 1[24$.

The repulsive image-charge interactions lead to depletion of ions from the dielectric interface of roughly a distance of $l_{B} / 2$, in the case of the planar geometry; this distance is approximately independent of the screening length. The effect of curvature is important when the radius of the particle becomes small compared to the screening length $\kappa R<1$. For decreasing values of $\kappa R$, the magnitude of the screened image interaction is lowered resulting in a decreased amount of ion desorption. As the radius of the sphere becomes greater than 


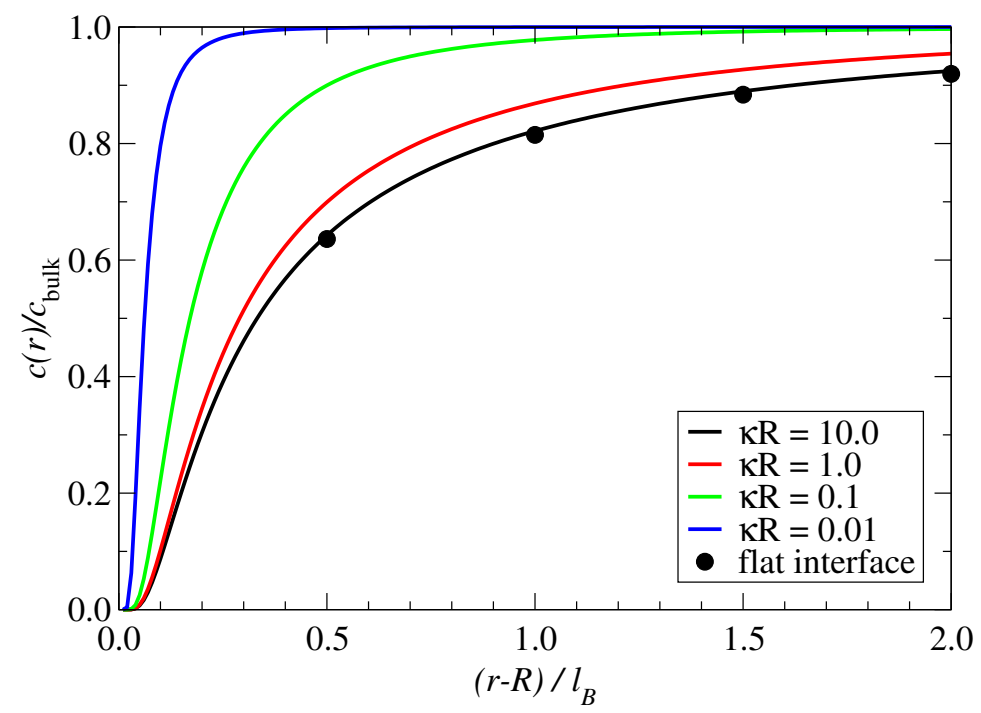

Figure 2: Density profile for symmetric electrolytes near a dielectric sphere with $\varepsilon^{\prime} / \varepsilon=0$, $\kappa l_{B}=0.1$, and: $\kappa R=0.01$ (blue line), $\kappa R=0.1$ (green line), $\kappa R=1$ (red line), $\kappa R=10$ (black), and planar interface (circles). plate.

\subsection{Influence on interfacial tension}

The depletion of co-solvents from a surface leads to an increase in the surface tension. For a system at a co-solvent concentration $c$ with a depletion length $l$, the increase in surface tension $\Delta \sigma$ can be estimated using a simple entropy argument to give

$$
\Delta \sigma=k_{B} T l c
$$

When the surface has a lower dielectric constant than the solvent, then ions with a valency $z_{\alpha}$ are repelled from the interface by a distance of roughly $z_{\alpha}^{2} \Delta l_{B} / 2$. The increase in surface tension is then given by

$$
\Delta \sigma=\frac{k_{B} T \Delta l_{B}}{2} \sum_{\alpha} c_{\alpha} z_{\alpha}^{2}
$$


where $c_{\alpha}$ is the concentration of ion species $\alpha$. Experimentally, this can be related to the surface tension increment $k_{E}$, which is defined as:

$$
k_{E}=\frac{\partial \sigma}{\partial c_{\alpha}} .
$$

Using this approach gives a surface tension increment for monovalent ions in water $k_{E} \approx 0.87 \mathrm{mN} \mathrm{m}^{-1} \mathrm{M}^{-1}$, which is similar to experimental values for many monovalent ions [27, although there are exceptions, the most notable being mixtures of asymmetric electrolytes. For electrolyte mixtures, the surface tension increment is typically equal to the sum of the $k_{E}$ for each type of ion, weighted linearly by the product of their concentration and valency. The depletion due to image-charge interactions suggests that the contributions should be instead weighted by the square of the ion valency.

\section{Interactions between dielectric particles}

\subsection{Two plate geometry}

Depletion of ions from a low-dielectric interface should give rise to an entropic depletion force between two low-dielectric surfaces when immersed in an electrolyte solution analogous to what happens for excluded-volume driven depletion. The electrostatic depletion force was first examined using two lowdielectric plates separated by a high dielectric solvent contains a dissolved electrolyte. Early Monte Carlo simulations [28, 29] that incorporated image charge forces observed the expulsion of ions from between the plates as their separation distance decreased. The ion depletion leads to an effective plate-plate attraction proportional to the osmotic pressure of the electrolyte solution. Even in the absence of dielectric interfaces, ions are repelled from the interface due to the lack of ionic screening near to the interface. This repulsion also leads to an attractive depletion force between plates, albeit much weaker than when image 


\subsection{Influence on dispersion forces}

Differences between the dielectric constant of colloidal particles and the solvent leads to a dispersion, or van der Waals, interaction between the particles. Within Lifshitz theory, the zero-frequency contribution to the dispersion interaction energy $F$ is related to the Green's function of the Poisson equation [31, 32, 33.

$$
F=\frac{k_{B} T}{2} \operatorname{Tr} \ln \frac{G}{G_{\text {free }}} .
$$

In classical treatments of colloidal interactions, such as DLVO theory, the dispersion forces are typically treated independently of the electrostatic interactions. However, ion motion can screen the low-frequency fluctuations in the electron density, altering the decay of the dispersion interaction [31. The dispersion interaction between two plates separated by an electrolyte-free medium is given by an attractive force, which varies as $p \propto 1 / d$ (where $d$ is the inter-plate separation). The presence of electrolytes screens the attractive force, such that the pressure has an exponential decay given by $p \propto e^{-\kappa d} / d$ [34, 35], where $\kappa^{-1}$ is the screening length for the electrolyte solution. The ability of ions to screen the dispersion force will be reduced when ions are depleted from between the lowdielectric surfaces, providing an additional mechanism by which ion depletion can lead to an increase in inter-plate attraction.

The effect of ion depletion on the dispersion force has been examined selfconsistently using the variational perturbation theory. The pressure between two plates immersed in an electrolyte solution is shown in Fig. 3, where the dielectric constant of the plates $\varepsilon^{\prime}$ is taken to be much lower than that of the intervening solvent $\varepsilon$. Predictions of the variational perturbation theory [24, 36] are compared to what happens when uniform screening of the dispersion interaction is assumed. According to Fig. 3 increasing the ion concentration weakens the inter-plate attraction due to screening of the van der Waals interaction. In the variational approach, the interaction is more attractive due to weaker screening effects from the layer of ion depletion about each of the plates.

The difference in the pressure calculated by the variational approach and 
the pressure of a screened van der Waals interaction [31, 35] is plotted in the inset to Fig. 3. The deviation between the two cases increases with increasing electrolyte concentration, while the screened van der Waals interaction by itself follows the opposite trend (i.e. the force becomes weaker with increasing bulk electrolyte concentration).

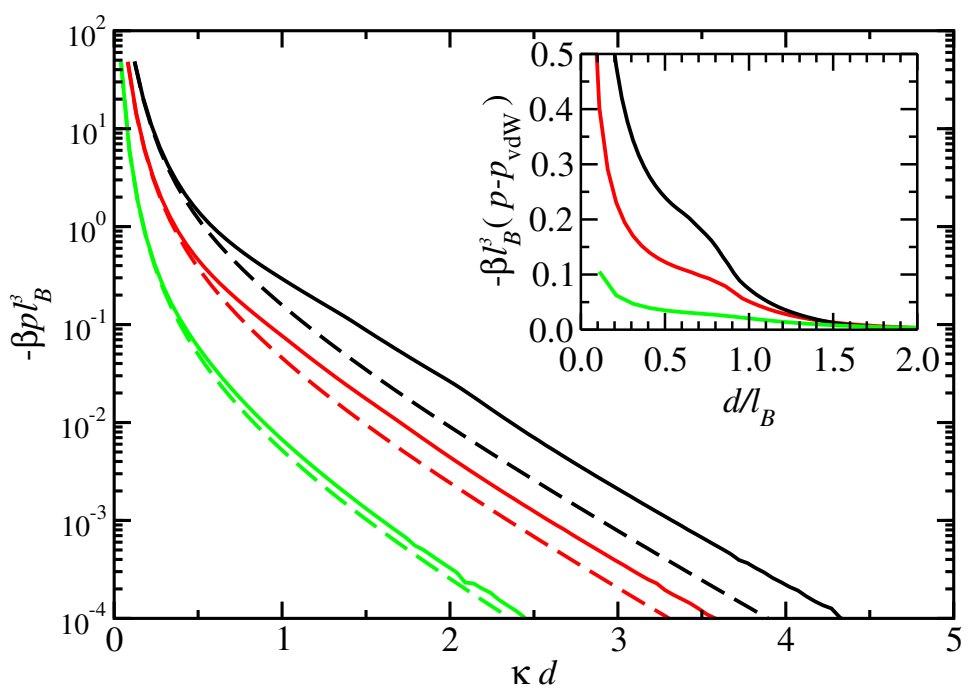

Figure 3: The pressure as a function of plate separation for $\eta=0$ and (i) $\kappa l_{B}=1.5$ (black), (ii) $\kappa l_{B}=1.0$ (red), and (iii) $\kappa l_{B}=0.5$ (green). The solid lines are the predictions of the variational perturbation theory, and the dashed lines are for the screened dispersion pressure, assuming a uniform screening length. The inset gives the deviation of the pressure from the screened dispersion pressure.

Figure 4 shows the effect of electrolyte concentration on the pressure between plates at a fixed separation. The results of the variational treatment are compared against the standard screened van der-Waals interaction. The difference between the pressures in the two cases can be attributed to the attractive osmotic depletion force and the change in the van der Waals interaction. For low bulk electrolyte concentrations $\kappa l_{\mathrm{B}} \ll 0.2$, the depletion-induced attraction is much smaller than the van der Waals interaction felt at the same distance. However, if the screening length becomes smaller than $l_{B}$ (e.g., at 
high electrolyte concentrations), the osmotic pressure force becomes comparable to the van der Waals interaction for plate-plate separations equal to or less than $l_{\mathrm{B}}$. Since the van der Waals interaction at $d=l_{B}$ is almost unaffected by the electrolytes, a good approximation for the magnitude of the osmotic depletion force at this separation is the difference between the total pressure (solid line in Fig. 45 and the van der Waals interaction in the absence of electrolytes $\left(-\beta l_{B}^{3} p_{\mathrm{vdW}}^{\kappa=0}\left(d=l_{B}\right)=0.0477\right)$. For values of $\kappa l_{\mathrm{B}}>0.5$, the magnitude of the osmotic depletion force becomes comparable to or greater than the unscreened dispersion force.

At plate separations larger than one Bjerrum length, electrolyte begins to enter the region between the plates and effectively screens the interaction. This can be seen by considering the case when $d=2 l_{B}$ in Fig. 4 where the interaction between the plates decreases with increasing electrolyte concentration (solid red line).

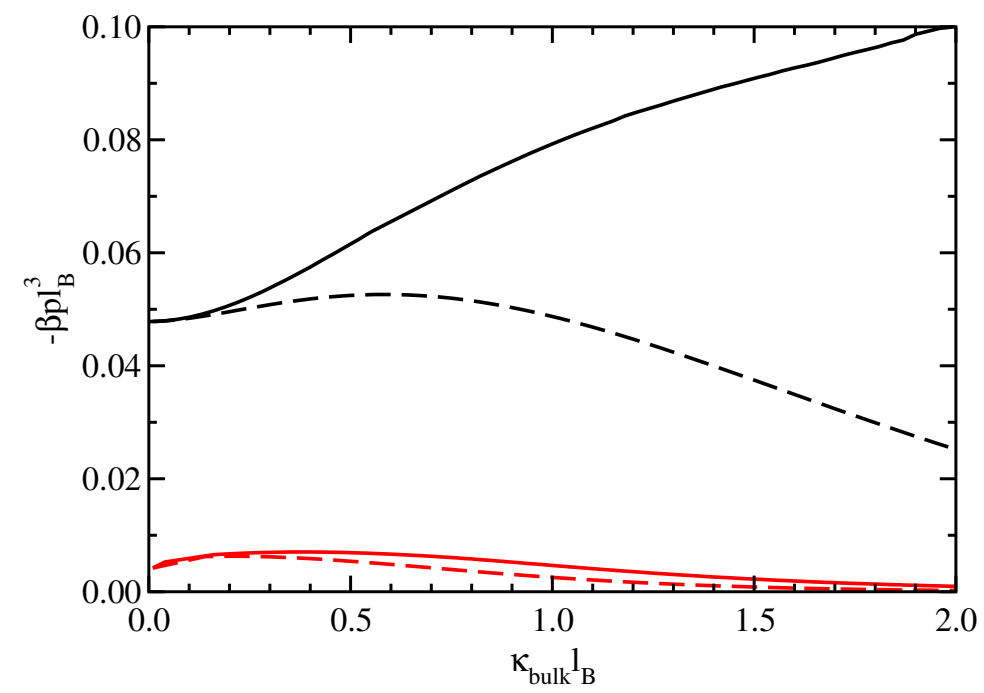

Figure 4: The variation of the pressure as a function of bulk screening length at constant plate-plate separation for $\eta=0$. (i) $d=l_{B}$ (black line), and (ii) $d=2 l_{B}$ (red line). The solid lines are the predictions of the variational perturbation theory, and the dashed lines are for the screened dispersion pressure. 
The electrostatic depletion force provides a partial explanation for the origin of salting-out interactions [37. Salting-out interactions are correlated with the preferential exclusion of the co-solvent molecules (or electrolytes) about the solute [38, 39]. The electrostatic depletion mechanism links the depletion of electrolyte about the solute to an attractive force between solute molecules. More importantly, salting-out interactions are not screened by increasing ionic strength, but rather increase in proportion to salt concentration. This behavior is accurately represented by the variational theory, in that the range of the interaction is independent of salt concentration, as it scales with the ion depletion layer thickness given by $l_{\mathrm{B}}$. Thus, similarly to the excluded volume force between colloids immersed in a polymer solution, the electrostatic depletion force has a constant range and a magnitude proportional to the osmotic pressure of the co-solvent (i.e. electrolyte). The attractive force becomes comparable to the dispersion force at $\kappa l_{\mathrm{B}} \sim 1.4$, corresponding to a concentration for a symmetric electrolyte mixture of approximately $1 \mathrm{M}$. This concentration is consistent with salting-out behavior, which is typically observed at salt concentrations greater than $1 \mathrm{M}$ [37]. A similar mechanism likely explains the increase of protein folding stability in concentrated salt solutions, which has been linked to image forces [40].

Accurate representation of electrostatic forces requires taking into account specific ion effects, which can arise from differences in the excess ion polarizability. A large excess ion polarizability is correlated with weak adsorption of ions to non-polar surfaces due to a combination of ion-surface dispersion and solvation forces [41, 42, 43]. These effects have been incorporated into electricdouble layer theories by including an ion-surface potential of mean force at the Poisson-Boltmzann level [44, 45, 46]. The approaches are able to capture the experimentally-observed reversal of the Hofmeister series for the saltingout effectiveness of charged, low-dielectric surfaces (such as proteins) [47]. An improved understanding requires combining the ion-specific effects self consistently with the image-charge force. Along these lines, recent work has included specific ion effects by the addition of a phenomenological adhesion parameter 
using a loop correction to the mean field theory [48]. Another interesting route has been to include the polarizability of the ions, which has been examined in the weak and strong coupling limits [49; this self-consistently incorporates not only the induced dipole interactions but also the dispersion forces between the ions and the ions and the dielectric interface.

\subsection{Other effects}

The magnitude of the image-charge interaction is proportional to the ion valency squared. For asymmetric electrolytes, the ion with the higher valency will be more strongly depleted from a low-dielectric interface. The depletion will cause an effective charge on the dielectric particle as lower valency ions will form a cloud about the colloids. This has implications for the interaction between low-dielectric particles in polyelectrolyte solutions.

The electrostatic depletion force not only occurs for ions, but also applies to neutral, multipolar co-solvents. The charge separation within a multipolar (e.g., dipolar) co-solvent will also lead to image-charge interactions with a dielectric interface. The magnitude of this interaction is weaker than that for a charged cosolvent because each of the charges within the multipolar molecule will interact with the image charges generated by the other charges. Consequently, for a low-dielectric interface there is a reduction in the net image-charge repulsion.

For multipolar molecules, the image-charge interactions will not only lead to a repulsion, but there is also a tendency to align molecules with the interface. Dipolar molecules will tend to align parallel to a low-dielectric interface.

225 This results in a different dielectric response of the solution parallel $\left(\varepsilon_{\|}\right)$and perpendicular $\left(\varepsilon_{\perp}\right)$ to the interface. [50, 51].

The addition of multipolar particles increases the dielectric constant of the solution, which will increase the attractive dispersion interactions between lowdielectric particles. However, the depletion of dipolar molecules from between the particles will decrease the dielectric constant of the intervening solution, thereby weakening the dispersion interaction. 


\section{Conclusions}

We have discussed image-charge interactions between dielectric interfaces and ions. For low-dielectric particles, this leads to an ion depletion layer which

235 sure of the bulk electrolyte solution and a reduction in the screening of the dispersion forces. This mechanism provides attractive force between low-dielectric particles which increases in magnitude with electrolyte concentration and has a relatively constant range (of about $l_{B}$ ). The electrostatic depletion force could 240 play a significant role in salting out of hydrophobic solutes and colloidal objects, such as proteins.

Interactions between colloids are sensitive to ion type, and several attempts have been made to theoretically capture these differences through incorporating specific ion-surface interactions or ion polarizability. It is expected that specific future direction for the investigation.

\section{References}

[1] S. Asakura, F. Oosawa, On interaction between two bodies immersed in a solution of macromolecules, J. Chem. Phys. 22 (7) (1954) 1255-1256. doi:10.1063/1.1740347.

[2] J. D. Jackson, Classical Electrodynamics, Wiley, New York, 1975.

[3] A. P. dos Santos, A. Bakhshandeh, Y. Levin, Effects of the dielectric discontinuity on the counterion distribution in a colloidal suspension, J. Chem. Phys. 135 (4) (2011) 044124. doi:10.1063/1.3615940.

[4] L. Lue, P. Linse, Macroion solutions in the cell model studied by field theory and monte carlo simulations, J. Chem. Phys. 135 (22) (2011) 224508.

doi:10.1063/1.3665450, 
[5] Z. Xu, Y. Liang, X. Xing, Mellin transform and image charge method for dielectric sphere in an electrolyte, SIAM Journal on Applied Mathematics 73 (4) (2013) 1396-1415. doi:10.1137/120894348.

[6] P. Linse, L. Lue, Green's function for a spherical dielectric discontinuity and its application to simulation, J. Chem. Phys. 140 (4) (2014) 044903. doi:10.1063/1.4862148,

[7] D. Boda, D. Gillespie, W. Nonner, D. Henderson, B. Eisenberg, Computing induced charges in inhomogeneous dielectric media: Application in a monte carlo simulation of complex ionic systems, Phys. Rev. E 69 (2004) 046702. doi:10.1103/PhysRevE.69.046702

[8] S. Tyagi, M. Süzen, M. Sega, M. Barbosa, S. S. Kantorovich, C. Holm, An iterative, fast, linear-scaling method for computing induced charges on arbitrary dielectric boundaries, J. Chem. Phys. 132 (15) (2010) 154112. doi:10.1063/1.3376011.

[9] V. Jadhao, F. J. Solis, M. O. de la Cruz, Simulation of charged systems in heterogeneous dielectric media via a true energy functional, Phys. Rev. Lett. 109 (2012) 223905. doi:10.1103/PhysRevLett.109.223905.

[10] V. Jadhao, F. J. Solis, M. Olvera de la Cruz, A variational formulation of electrostatics in a medium with spatially varying dielectric permittivity, J. Chem. Phys. 138 (5) (2013) 054119. doi:10.1063/1.4789955.

[11] F. Fahrenberger, Z. Xu, C. Holm, Simulation of electric double layers around charged colloids in aqueous solution of variable permittivity, J. Chem. Phys. 141 (6) (2014) 064902. doi:10.1063/1.4892413.

[12] Z. Xu, A fast algorithm for treating dielectric discontinuities in charged spherical colloids, Interdiscip. Sci. Comput. Life Sci. 4 (2012) 19-26. doi: 10.1007/s12539-012-0113-1. 
[13] Z. Xu, Electrostatic interaction in the presence of dielectric interfaces and polarization-induced like-charge attraction, Phys. Rev. E 87 (2013) 013307. doi:10.1103/PhysRevE.87.013307

[14] C. Wagner, Phys. Z. 25 (1924) 474.

[15] L. Onsager, N. N. T. Samaras, The surface tension of debyehckel electrolytes, J. Chem. Phys. 2 (8) (1934) 528-536. doi:10.1063/1.1749522.

[16] T. Croxton, D. A. McQuarrie, G. N. Patey, G. M. Torrie, J. P. Valleau, Ionic solution near an uncharged surface with image forces, Can. J. Chem. 59 (13) (1981) 1998-2003. doi:10.1139/v81-295.

[17] J.-N. Aqua, F. Cornu, Density profiles in a classical coulomb fluid near a dielectric wall. i. mean-field scheme, J. Stat. Phys. 105 (1/2) (2001) 211243.

[18] J.-N. Aqua, F. Cornu, Density profiles in a classical coulomb fluid near a dielectric wall. ii. weak-coupling systematic expansions, J. Stat. Phys. $105(1 / 2)(2001) 245-283$.

[19] P. Attard, D. J. Mitchell, B. W. Ninham, Beyond Poisson-Boltzmann: Images and correlations in the electric double layer. I. Counterions only, J. Chem. Phys. 88 (8) (1988) 4987-4996.

[20] P. Attard, D. J. Mitchell, B. W. Ninham, Beyond Poisson-Boltzmann: Images and correlations in the electric double layer. II. Symmetric electrolyte, J. Chem. Phys. 89 (7) (1988) 4358-4367.

[21] R. D. Coalson, A. Duncan, Systematic ionic screening theory of macroions, J. Chem. Phys. 97 (8) (1992) 5653-5661.

[22] R. R. Netz, Debye-Hückel theory for interfacial geometries, Phys. Rev. E 60 (3) (1999) 3174-3182.

[23] D. S. Dean, R. R. Horgan, Field theoretic calculation of the surface tension for a model electrolyte system, Phys. Rev. E 69 (6) (2004) 061603. 
[24] R. A. Curtis, L. Lue, Electrolytes at spherical dielectric interfaces, J. Chem. Phys. 123 (2005) 174702. doi:10.1063/1.2102890.

[25] L. Lue, A variational field theory for solutions of charged, rigid particles, Fluid Phase Equil. 241 (2006) 236-247. doi:10.1016/j.fluid.2005.11. 007.

[26] M. Kanduc, R. Podgornik, Electrostatic image effects for counterions between charged planar walls, Euro. Phys. J. E 23 (2007) 265-274.

[27] Y. Marcus, Individual ionic surface tension increments in aqueous solutions, Langmuir 29 (9) (2013) 2881-2888. doi:10.1021/1a3041659.

[28] L. Guldbrand, B. Jönsson, H. Wennerström, P. Linse, Electrical double layer forces. a monte carlo study, J. Chem. Phys. 80 (5) (1984) 2221-2228. doi:10.1063/1.446912,

[29] D. Bratko, B. Jönsson, H. Wennerström, Electrical double-layer interactions with image charges, Chem. Phys. Lett. 128 (5-6) (1986) 449-454. doi:10.1016/0009-2614(86)80652-2.

[30] D. Bratko, D. Henderson, Osmotic interactions between neutral surfaces in an electrolyte solution, Phys. Rev. E 49 (1994) 4140-4144. doi:10.1103/ PhysRevE.49.4140.

[31] J. Mahanty, B. W. Ninham, Dispersion Forces, Academic Press, London, 330 1976.

[32] V. A. Parsegian, Van der Waals Forces A Handbook for Biologists, Chemists, Engineers, and Physicists, Cambridge, 2006.

[33] M. M. Hatlo, L. Lue, The role of image charges in the interactions be口 tween colloidal particles, Soft Matter 4 (2008) 1582-1596. doi:10.1039/ B803783C,

[34] R. R. Netz, Debye-Hückel theory for slab geometries, Eur. Phys. J. E 3 (2) (2000) 131-141. 
[35] R. R. Netz, Static van der Waals interactions in electrolytes, Eur. Phys. J. E 5 (2001) 189-205.

₫ planar surfaces, J. Chem. Phys. 128 (16) (2008) 164717. doi:10.1063/1. 2908738

[37] R. A. Curtis, L. Lue, A molecular approach to bioseparations: Connecting protein-protein and protein-solvent interactions, Chem. Eng. Sci. 61 (2006)

345 907-923. doi:10.1016/j.ces.2005.04.007.

[38] T. Arakawa, S. N. Timasheff, Preferential interactions of proteins with salts in concentrated-solutions, Biochemistry 21 (25) (1982) 6545-6552.

[39] T. Arakawa, S. N. Timasheff, Mechanism of protein salting in and salting out by divalent-cation salts - balance between hydration and salt binding, Biochemistry 23 (25) (1984) 5912-5923.

[40] D. L. Beauchamp, M. Khajehpour, Studying salt effects on protein stability using ribonuclease t1 as a model system, Biophys. Chem. 161 (2012) 29-38.

[41] P. Jungwirth, D. J. Tobias, Ions at the air/water interface, J. Phys. Chem. B 106 (25) (2002) 6361-6373.

[42] P. Jungwirth, D. J. Tobias, Specific ion effects at the air/water interface, Chemical Reviews 106 (4) (2006) 1259-1281.

[43] D. Horinek, A. Herz, L. Vrbka, F. Sedlmeier, S. I. Mamatkulov, R. R. Netz, Specific ion adsorption at the air/water interface: The role of hydrophobic solvation, Chemical Physics Letters 479 (4-6) (2009) 173-183.

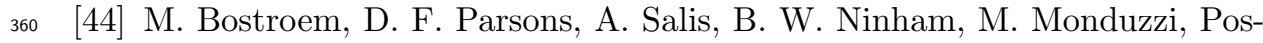
sible origin of the inverse and direct hofmeister series for lysozyme at low and high salt concentrations, Langmuir 27 (15) (2011) 9504-9511. 
[45] N. Schwierz, D. Horinek, R. R. Netz, Reversed anionic hofmeister series: The interplay of surface charge and surface polarity, Langmuir 26 (10) (2010) 7370-7379. doi:10.1021/la904397v.

[46] N. Schwierz, D. Horinek, R. R. Netz, Anionic and cationic hofmeister effects on hydrophobic and hydrophilic surfaces, Langmuir 29 (8) (2013) 26022614.

[47] Y. J. Zhang, P. S. Cremer, The inverse and direct hofmeister series for lysozyme, Proc. Natl. Acad. Sci. U.S.A 106 (36) (2009) 15249-15253.

[48] T. Markovich, D. Andelman, R. Podgornik, Surface tension of electrolyte solutions: A self-consistent theory, EPL (Europhysics Letters) 106 (1) (2014) 16002.

[49] V. Demery, D. S. Dean, R. Podgornik, Electrostatic interactions mediated by polarizable counterions: Weak and strong coupling limits, J. Chem. Phys. 137 (17) (2012) 174903.

[50] M. Kanduč, A. Naji, Y. S. Jho, P. A. Pincus, R. Podgornik, The role of multipoles in counterion-mediated interactions between charged surfaces: strong and weak coupling, J. Phys. Cond. Matt. 21 (42) (2009) 424103.

380 [51] S. Buyukdagli, R. Blossey, Dipolar correlations in structured solvents under 口 nanoconfinement, J. Chem. Phys. 140 (23) (2014) 234903. doi:10.1063/ 1.4881604 . 